\title{
Criminologie
}

\section{Trajectoires sociocarcérales des femmes autochtones au Québec : effets de l'incarcération sur l'exclusion sociale}

\section{Renée Brassard et Joane Martel}

Volume 42, numéro 2, automne-hiver 2009

Régulations sociopénales et peuples autochtones

URI : https://id.erudit.org/iderudit/038602ar

DOI : https://doi.org/10.7202/038602ar

Aller au sommaire du numéro

\section{Éditeur(s)}

Les Presses de l'Université de Montréal

ISSN

0316-0041 (imprimé)

1492-1367 (numérique)

Découvrir la revue

Citer cet article

Brassard, R. \& Martel, J. (2009). Trajectoires sociocarcérales des femmes autochtones au Québec : effets de l'incarcération sur l'exclusion sociale. Criminologie, 42(2), 121-152. https://doi.org/10.7202/038602ar
Résumé de l'article

Le présent article expose les résultats d'une étude qualitative portant sur les effets de l'incarcération provinciale sur la trajectoire de vie de sept femmes autochtones au Québec. Au début du cycle de vie, trois répondantes évoluent dans un milieu familial relativement stable, quatre vivent dans un milieu familial connaissant de multiples problèmes sociaux. Ces distinctions se maintiennent pratiquement jusqu'aux expériences de détention, mais s'estompent considérablement après le passage en détention. Le stigmate du casier judiciaire et les règles d'accès aux programmes dits de réinsertion sociale sont les effets égalisateurs les plus perceptibles des conditions de vie de notre groupe de répondantes. Ils contribuent à niveler vers le « bas " les conditions de vie des interviewées. Ainsi, le casier judiciaire contribue à exclure définitivement les répondantes du marché de l'emploi, rend plus complexe l'accès au logement et provoque la marginalisation des femmes dans leur communauté d'origine. Le poids de l'incarcération est donc important dans la mise en marge des femmes autochtones. Cependant, il doit être relativisé dans la mesure où il s'ajoute à des conditions et à un contexte de vie qui précède la prise en charge pénale. 


\title{
Trajectoires sociocarcérales des femmes autochtones au Québec: effets de l'incarcération sur l'exclusion sociale
}

\author{
Renée Brassard \\ Professeure agrégée \\ École de service social, Université Laval \\ Chercheure associée \\ Centre international de criminologie comparée, Université de Montréal \\ renee.brassard@svs.ulaval.ca \\ Joane Martel \\ Professeure titulaire \\ École de service social, Université Laval \\ joan.martel@svs.ulaval.ca
}

RÉSUMÉ - Le présent article expose les résultats d'une étude qualitative portant sur les effets de l'incarcération provinciale sur la trajectoire de vie de sept femmes autochtones au Québec. Au début du cycle de vie, trois répondantes évoluent dans un milieu familial relativement stable, quatre vivent dans un milieu familial connaissant de multiples problèmes sociaux. Ces distinctions se maintiennent pratiquement jusqu'aux expériences de détention, mais s'estompent considérablement après le passage en détention. Le stigmate du casier judiciaire et les règles d'accès aux programmes dits de réinsertion sociale sont les effets égalisateurs les plus perceptibles des conditions de vie de notre groupe de répondantes. Ils contribuent à niveler vers le "bas" les conditions de vie des interviewées. Ainsi, le casier judiciaire contribue à exclure définitivement les répondantes du marché de l'emploi, rend plus complexe l'accès au logement et provoque la marginalisation des femmes dans leur communauté d'origine. Le poids de l'incarcération est donc important dans la mise en marge des femmes autochtones. Cependant, il doit être relativisé dans la mesure où il s'ajoute à des conditions et à un contexte de vie qui précède la prise en charge pénale.

MOTS-CLÉs - Autochtone, exclusion, femmes, prison, Québec, réinsertion, trajectoire de vie. 


\section{Introduction}

Des quelques études qui se sont intéressées, en tout ou en partie, à l'incarcération des femmes autochtones ${ }^{1}$, il ressort que ces dernières constituent un groupe particulièrement exclu et marginalisé (Zambrowski, 1986; Sugar et Fox, 1990; Grosbsmith, 1995; Azaola, 1996; La Prairie, 1996; Ross, 1998; Brassard, 2006). Les plus récents profils sociodémographiques des femmes autochtones incarcérées au Canada mettent en évidence des antécédents d'abus d'alcool et/ou de drogues, une situation socio-économique fragilisée, une sous-scolarisation chronique, un passé de violence et de survictimisation et, enfin, des contacts récurrents avec l'institution carcérale (LaPrairie, 1997; Canada, 2001). Bref, les femmes autochtones qui sont détenues dans les institutions carcérales du Canada ont souvent cette caractéristique commune d'être exposées à des itinéraires de vie conduisant fréquemment à l'exclusion sociale.

L'intérêt scientifique pour la thématique de l'exclusion sociale, au cours des vingt dernières années à tout le moins, a conduit certains chercheurs à investiguer la prison et à documenter, à partir de trajectoires de vie, les manières dont l'incarcération crée des ruptures avec les espaces d'insertion sociale (Laberge et al., 1998; Chantraine, 2004) et produit des effets néfastes chez des groupes particulièrement fragilisés (Landreville et al., 1981; Hamelin, 1989). Or, malgré que les femmes autochtones incarcérées au Canada, comme au Québec, constituent nettement un des groupes sociaux les plus fragilisés, aucune étude empirique n'a encore tenté de comprendre les effets de l'incarcération sous l'angle des trajectoires de vie des femmes autochtones et, encore moins, dans la perspective théorique de l'exclusion sociale. Cet article vise précisément à comprendre dans quelle mesure l'expérience carcérale

1. En vertu de la Loi constitutionnelle canadienne de 1982, le terme «Autochtone» désigne tous les premiers habitants du Canada et leurs descendants. Le gouvernement canadien reconnaît trois catégories officielles d'Autochtones: les Amérindiens, les Inuits et les Métis. Selon les données du Recensement de 2006 (Statistique Canada, 2008), le nombre de personnes qui se sont identifiées comme Autochtones au Canada atteint 1172 790. En 2006, les Amérindiens, les Métis et les Inuits, comptaient pour près de $4 \%$ de la population totale du Canada. On compte actuellement 615 collectivités des Premières Nations et 53 collectivités inuites réparties à travers le Canada (AINC, 2008). Sur le plan international, le Canada se classait au deuxième rang pour ce qui est de la proportion d'Autochtones au sein de la population, derrière la Nouvelle-Zélande, où les Maoris formaient $15 \%$ de la population. Selon les données du recensement de 2006, le Canada compte un peu moins d'un demi-million de femmes autochtones (Statistique Canada, 2008). Cette population représentait $3 \%$ de toute la population féminine canadienne. 
renforce, neutralise ou, encore, atténue le processus d'exclusion sociale des femmes autochtones. Afin de répondre à ce questionnement, différents segments de vie des femmes autochtones, ainsi que certains moments-clés de leurs trajectoires pré-carcérales, carcérales et postcarcérales sont intégrés et mis en rapport les uns avec les autres. Plus précisément, il est question de mettre en lumière les liens structurels pouvant amarrer entre eux les conditions actuelles de vie des femmes autochtones incarcérées, les conditions de leurs milieux d'origine, les éléments qui précipitent leur contact avec le système de justice pénale, leur expérience carcérale et, enfin les effets de cette incarcération sur leur trajectoire de vie respective.

\section{Écrits scientifiques sur les effets de l'incarcération}

Le récent manuel dirigé par Liebling et Maruna (2005) et intitulé The Effects of Imprisonment expose de manière détaillée l'émergence de l'intérêt scientifique entourant les effets de l'incarcération, ainsi que les débats actuels que ces effets suscitent toujours aujourd'hui. Plus particulièrement, Liebling et Maruna nous informent que l'un des postulats fondamentaux sur lesquels repose une part importante des études qui abordent les effets de l'incarcération est que l'incarcération produit incontestablement des effets dans la vie des individus. À l'instar d'une telle prémisse, de nombreuses recherches, surtout quantitatives, ont mis en évidence, au cours des cinq dernières décennies, un ensemble d'effets adverses que l'incarcération serait censée produire (Grassian, 1983; Grobsmith, 1995). Les complexités méthodologique et conceptuelle rattachées à la démonstration efficiente d'effets de l'incarcération ont, toutefois, rapidement obligé la recherche à examiner davantage les moments-clés et les critères de plus en plus précis qui peuvent influencer la nature et la portée des effets de l'incarcération. Cette constatation a donné lieu à une fragmentation des écrits scientifiques destinés aux effets de l'incarcération. D’une part, certains font aujourd'hui état des effets directs de l'incarcération, par exemple sur la santé physique ou psychologique des détenus (Ditton, 1999) ou, encore, sur la consommation de psychotropes (Plourde et al., 2007). D'autres portent plutôt leur attention sur les effets indirects que peut produire l'incarcération sur les différentes sphères de la vie des personnes incarcérées telles que la vie familiale (Ferraro et al., 1983; Travis et Waul, 2003) ou, encore, l'emploi (Metcalf et al., 2001; Braman, 2004; Liebling et Maruna, 
2005). Les connaissances actuelles commandent également que les effets de l'incarcération soient distingués selon le moment où ceux-ci se produisent, soit les effets se manifestant pendant la période carcérale (ex. : transmission du VIH, stratégies d'adaptation) ou au cours de la période post-carcérale (ex. : casier judiciaire, reprise des liens familiaux) (Petersilia, 2003; Maruna et Immarigeon, 2004). Cette importante distinction conceptuelle a été utilisée dans le cadre de notre étude, ce qui, de concert avec l'utilisation de trajectoires de vie, nous a permis de développer un argumentaire analytique qui intègre les phases précarcérale, carcérale et post-carcérale.

Outre la temporalisation analytique qui s'est opérée entre les effets qui se manifestent pendant et après la détention, d'autres critères de différenciation des effets de l'incarcération ont aussi été mis en lumière par la recherche, notamment, la classe sociale (Landreville et al., 1981), le genre (Frigon et Kérisit, 2000) et la culture (Sloop, 2006), qui figurent parmi les premiers critères de différenciation documentés. Ces travaux ont le mérite particulier d'avoir permis de constater que les effets produits par l'incarcération sont davantage considérables auprès des populations les plus exclues de nos sociétés, que l'on pense notamment aux mères incarcérées (Woolredge et Masters, 1993; Kauffman, 2001; Blanchard, 2002), aux personnes itinérantes (Laberge et al., 1998) ou aux immigrants (Joly et Beckford, 2006). Dans cette lignée, l'étude qualitative des coûts sociaux du système de justice pénale de Landreville et al. (1981), réalisée auprès de trois groupes de participants issus de différentes classes sociales ayant connu des démêlés avec la justice, a spécialement démontré que la classe sociale a un impact déterminant sur les mécanismes de production et de neutralisation des effets. Quelques années plus tard, Laberge et al. (1998) réitèrent ce constat dans une étude qui explore les trajectoires de vie de 17 itinérants de Montréal ayant été incarcérés. Les résultats suggèrent que, par les effets qu'elle produit auprès de cette population vulnérabilisée (la perte des quelques biens matériels qu'ils possèdent, le non-accès à un logement décent, etc.), la prison joue un rôle de premier plan dans la production de l'exclusion sociale et de l'itinérance. Nul doute, ce type d'études a résolument contribué à donner une voix à certains groupes minoritaires et marginalisés. Cependant, d'autres groupes vulnérabilisés semblent avoir moins retenu l'attention scientifique. C'est le cas, notamment, des femmes autochtones qui sont reconnues depuis plus de trente ans (Brassard, 2006) comme une population sujette à l'exclusion sociale. 
Or, malgré ce constat désolant, les femmes autochtones continuent d'être ignorées dans les travaux de recherche sur les effets de l'incarcération. Même si les femmes autochtones ont été sollicitées à plusieurs reprises dans le cadre de différentes enquêtes de terrain de même que lors de commissions d'enquête canadiennes traitant de la question de la justice et des Autochtones (Sugar et Fox, 1990; Commission royale sur les peuples autochtones, 1996; Monture-Angus, 2003), on connaît encore mal les effets de l'incarcération qui les affligent.

Nous avons recensé moins d'une dizaine d'études, dont une seule étude canadienne, traitant de la question des effets de l'incarcération chez les Autochtones. Pour la majorité, ces études traitent des effets de l'incarcération durant la phase carcérale. À titre d'exemple, une étude ethnographique réalisée au sein d'une institution carcérale pour femmes située dans l'État du Montana montre comment l'incarcération massive, et souvent récurrente, des femmes autochtones amène les détenues à se reconnaître entre elles, dans un premier temps, et à se regrouper, dans un second temps. Ces regroupements tendent à être réinterprétés dans une logique proprement carcérale - notamment en matière de risque - et produisent ainsi des effets négatifs dans la trajectoire carcérale des femmes autochtones (Ross, 1998). Une telle perception de ces regroupements comme une menace inciterait le personnel correctionnel à échafauder différentes stratégies visant à briser la cohésion entre les détenues autochtones, tel le transfert vers d'autres institutions de détention.

Certaines de ces études ont, par ailleurs, révélé que l'incarcération provoque des ruptures importantes avec la sphère familiale (Trevethan, Auger, Moore, MacDonald et Sinclair, 2002), la communauté d'origine ainsi que la culture autochtone, qui sont, toutes trois, des composantes marquantes de l'identité autochtone. Grossman (1992) indique, à cet égard, que ces types de rupture sont singulièrement plus néfastes pour les femmes autochtones incarcérées. Souvent incarcérées dans des institutions spatialement éloignées de leurs enfants et de leur communauté, celles-ci sont privées de contacts avec leurs familles, leurs enfants et leur culture.

Quelques autres études permettent de constater que, au-delà de la phase carcérale, les effets de l'incarcération tendent aussi à dépasser les murs de la prison. À partir d'une analyse autobiographique de son propre parcours carcéral, Morin (2002) a observé que les valeurs et les attitudes proprement carcérales tendent à être exportées au sein des communautés autochtones, exposant ainsi les jeunes Autochtones aux 
valeurs carcérales à travers leurs relations sociales et rendant alors possible la transmission et la reproduction intergénérationnelles de telles valeurs.

Au-delà des effets qui se manifestent durant les phases carcérale et post-carcérale, les constats évoqués par certaines auteures nous ont convaincues que les interprétations analytiques des effets de l'incarcération chez les femmes autochtones pouvaient varier selon que l'on prend en compte ou non les conditions de vie en amont des expériences carcérales: "For Aboriginal women, prison is an extension of life on the outside, and because of this it is impossible for us to heal there [...]» (Sugar et Fox, 1990: 476-477). Quelques années plus tard, Grobsmith (1995: 7) abondera dans un sens similaire en arguant que:

In the majority of cases [of Native prisoners], there were no major changes in lifestyle nor abandonment of youtbful self-destructive behavior [upon release from prison]. [...] the return to drinking, the revocation of parole status, and the return to incarceration were all depressing realities [...].

De tels constats permettent de lever partiellement le voile sur une réalité davantage occultée dans la littérature puisque les études qui abordent les effets de l'incarcération ont, jusqu'à maintenant, surinvesti les phases carcérale et post-carcérale au détriment d'une appréhension plus systématique du caractère dynamique et évolutif des conditions de vie des femmes autochtones incarcérées. Or, pour saisir ce caractère évolutif des conditions de vie, il convient, d'entrée de jeu, de les (ré)insérer dans la trame narrative que les femmes autochtones construisent à l'égard de leur propre trajectoire biographique. C'est dans cette ligne de pensée que la prochaine section présente la population à l'étude.

\section{Les femmes autochtones incarcérées au Canada: portrait d'une population exclue}

Les données canadiennes montrent depuis déjà quelques années que la surreprésentation des femmes autochtones dans les institutions carcérales est proportionnellement plus élevée que celle des hommes autochtones (Brassard, 2006). Alors que les personnes d'origine autochtone représentent $4 \%$ de la population canadienne en général (Canada, 2008), les femmes autochtones comptent pour près du quart $(23 \%)$ de l'ensemble de la population carcérale féminine au Canada; les hommes autochtones représentant, pour leur part, $18 \%$ des admissions dans les établissements de détention fédéraux (Canada, 2004) 
À la fin de septembre 2007, 32\% des femmes qui purgeaient une peine fédérale de détention au Canada étaient d'origine autochtone. Outre le fait alarmant que le nombre de personnes autochtones détenues dans des établissements fédéraux canadiens se soit accru de $26,4 \%$ entre 1997 et 2007, le nombre de femmes autochtones aurait atteint une augmentation ahurissante de $151 \%$ au cours de la même période (Enquêteur correctionnel, 2007).

Ces chiffres sont d'autant plus préoccupants que différentes études qui mettent en lumière les conditions de vie des femmes autochtones incarcérées indiquent que leurs conditions de vie avant leur incarcération et au moment où elles purgent leur sentence carcérale (et ce, tant dans les établissements provinciaux que fédéraux) sont des plus précaires. Alors que l'étude de Dubec (1982) constitue la première recherche canadienne à s'être consacrée à la situation des femmes autochtones au sein des établissements de détention fédéraux au Canada, il faudra attendre le début des années 1990 pour qu'une seconde étude, celle de Sugar et Fox (1990), pousse davantage la réflexion. L'étude de Sugar et Fox avait été entreprise auprès de 39 femmes autochtones qui purgeaient une sentence fédérale. Elle révéla, par exemple, que près de $90 \%$ des femmes autochtones de leur échantillon avaient été abusées sexuellement et/ou violentées physiquement durant leur enfance et que la grande majorité des participantes, soit 34 sur 39, avaient été victimes de violence conjugale à l'âge adulte. Depuis l'étude de Sugar et Fox (1990), de nombreuses recherches ont souligné non seulement la victimisation des femmes autochtones incarcérées, mais également le fait que cette victimisation constituait l'une des caractéristiques marquantes de la vie pré-carcérale des femmes autochtones au Canada. Outre la mise en lumière de cette caractéristique, Sugar et Fox (1990) ont aussi mis en évidence les nombreux placements institutionnels quavaient connus les femmes autochtones au cours de leur adolescence. De l'ensemble des femmes autochtones interrogées, 14 avaient connu des placements en foyer nourricier ou dans des milieux de prise en charge.

Par ailleurs, plusieurs chercheurs s'entendent sur le fait que la majorité des femmes autochtones incarcérées sont aux prises avec un problème de surconsommation d'alcool et que celui-ci joue un rôle important dans la commission d'infractions (Jaccoud, 1992; LaPrairie, 1996; Jackson, 1999 ; Brassard, 2006). À ces conditions de vie déjà difficiles s'ajoute une précarité socio-économique importante, notamment sur le plan des taux de prestation d'assurance-emploi et d'aide sociale qui, chez les 
femmes autochtones incarcérées, seraient jusqu'à huit fois plus élevés que dans la population en général (Johnson, 1986). D’autres études mettent en exergue les conditions de santé fragile des femmes autochtones incarcérées (Kreig, 2006).

Un sondage canadien réalisé par le Service correctionnel du Canada (Canada, 2001) indique qu'au moment de leur libération, 14 des 16 femmes autochtones échantillonnées étaient célibataires et cinq étaient les premières responsables de la garde de leurs enfants. Le document révèle également que $88 \%$ des femmes autochtones visées par ce sondage vivaient en milieu urbain et la plupart habitaient un logement non subventionné. Les données sur la situation d'emploi montrent qu'une seule participante occupait un emploi stable, alors que 15 autres étaient soit à la maison, soit sans travail, soit aux études. Ainsi, la plupart (82\%) d'entre elles étaient bénéficiaires de prestations d'aide sociale. En ce qui concerne le niveau de scolarisation, 14 des femmes autochtones interrogées avaient complété, au mieux, une cinquième secondaire.

Au Québec, les travaux de Jaccoud (1992) sur les admissions des Autochtones au sein des établissements de détention ont aussi montré que les femmes autochtones y étaient surreprésentées, mais de façon minime. Dans son étude, Jaccoud (1992) rapporte que durant cette période, les Amérindiennes représentaient 2,3\% des admissions dans les établissements de détention provinciaux au Québec, alors que cette population constituait $0,61 \%$ de la population générale de la province. Outre les travaux de Zambrowski-Cross et ceux de Brassard (2006), aucune autre étude ne s'est intéressée à la situation des femmes autochtones incarcérées au Québec. Zambrowski-Cross (1986) a mené son étude auprès de femmes autochtones ayant eu ou risquant d'avoir des démêlés avec le système de justice pénale dans la région de Montréal. À l'instar des résultats du sondage effectué par le Service correctionnel du Canada en 2001, la recherche de Brassard (2006) a également mis en évidence les conditions socioéconomiques précaires des femmes autochtones. Ainsi, on y apprend que la très grande majorité des femmes autochtones interrogées étaient prestataires de l'aide sociale et plus du trois quarts avaient complété moins d'une cinquième secondaire. Qu'elles soient canadiennes ou québécoises, ces données témoignent de l'exclusion des femmes autochtones incarcérées. 


\section{Cadre théorique et méthodologique}

L'exclusion sociale est considérée comme un processus dynamique et non pas comme un état (Castel, 1994; Gaugelac et Taboada Léonetti, 1994). Elle est envisagée comme le produit d'interactions entre les acteurs et les systèmes (Giddens, 1984). Tout au long de leur parcours de vie, les acteurs sociaux traversent différents espaces de vie (famille, travail, éducation, réseau relationnel) ${ }^{2}$ au sein desquels se produisent des interactions et des événements (Castel, 1994; McAll, 1995). L'exclusion se conceptualise comme le produit d'une série de ruptures ou de non-insertion dans les principaux espaces normatifs d'intégration tels que la famille, l'éducation et le travail (Castel, 1994; Paugam, 1996; Xiberras, 1998). À l'instar de Bourdieu (1979), nous considérons que les acteurs ne se déplacent pas au hasard dans l'espace et dans le temps. La trajectoire de vie des acteurs sociaux est influencée par la rencontre d'autres acteurs ou institutions, ou encore par leur non-rencontre. Nous posons d'entrée de jeu que les trajectoires de vie des individus sont construites par les intentions des acteurs sociaux, des acteurs interagissant selon leur appartenance multicatégorielle, et leur capital hérité (Bourdieu, 1979). La position de l'acteur social que nous privilégions est celle proposée, entre autres, par Dubet (1994), selon laquelle l'acteur social n'est pas totalement aveuglé par le poids des structures qu'il traverse, ni totalement clairvoyant et rationnel. Cette position a l'avantage de permettre de comprendre la logique d'action, la marge de manœuvre et les stratégies que les individus déploient au sein des structures sociales. L'exclusion sociale résulte d'un ensemble de ruptures, lesquelles contribuent à mettre des groupes sociaux à l'écart de la production des espaces de vie sociale active (travail, famille, éducation, loisirs, etc.) (Castel, 1994; McAll, 1995) et des pratiques communes de consommation et d'échanges (Paugam, 1996). Les acteurs n'ont donc plus accès aux formes de sociabilité, sauf celles que procurent les services d'aide et d'assistance. À la lumière des théories de l'exclusion sociale, il convient de considérer le milieu carcéral comme un territoire institutionnel au sein duquel se produisent des interactions et des

2. Bien que le mode d'intelligibilité qui découle du cadre théorique présente les espaces de vie d'une façon distincte et séparée, il importe de noter que ces espaces sont constamment en interaction les uns avec les autres. En ce sens, les interactions, les événements et les ruptures qui se produisent au sein d'un espace de vie ont des effets sur les autres espaces de vie des acteurs sociaux. 
événements et à l'intérieur duquel les femmes autochtones se déplaceront à travers différents espaces de vie comme l'emploi carcéral, la programmation institutionnelle ou encore le processus éducationnel tel qu'il est dispensé par la prison.

C'est en regard de cette position épistémologique et de nos objectifs de recherche que le récit de vie, défini comme l'histoire d'une vie, ou d'un segment de vie, telle que racontée par la personne qui l'a vécu (Denzin, 1970; Corbin et Strauss, 1990), devient un outil méthodologique approprié pour comprendre les effets de l'institution d'enfermement dans la trajectoire de vie des femmes autochtones. Le récit de vie est reconnu pour sa capacité d'appréhender, d'une part, les ruptures avec les espaces d'intégration sociale et, d'autre part, les événements marquants de l'expérience de vie des participants (Marchetti, 1996). Soulignons, par ailleurs, l'utilité indéniable du récit de vie pour appréhender les ruptures provoquées par des forces extérieures, de même que pour saisir la linéarité et/ou les mouvements circulaires des trajectoires de vie. Kellerhals et al. (1983) ajoutent que le récit de vie trouve toute sa richesse en ceci qu'il permet au chercheur de suivre la trajectoire des individus dans sa temporalité et son processus, permettant, par le fait même, de saisir le point de départ (la naissance) ainsi que le point d'arrivée d'une trajectoire de vie (le moment présent). Il constitue un outil privilégié pour capter le sens que les acteurs donnent à leur vie, à leurs actions et à leurs pratiques sociales (Bertaux, 1988; Lainé, 1998). Selon cette approche, l'acteur social devient porteur de structures sociales tout autant que participant à leur production (Balan et Jelin, 1980). Dans notre étude, les récits de vie de sept femmes autochtones ont été reconstruits dans le cadre de quatre à sept entrevues d'une durée d'une heure trente à deux heures chacune. Au total, 43 entrevues biographiques ont été réalisées auprès de sept répondantes autochtones. Les interviewées ont été recrutées dans les prisons pour femmes du Québec de même que dans les rues de Montréal. En vue de rendre compte de l'expérience de l'enfermement et de ses effets sur les trajectoires de vie des femmes autochtones, différents thèmes ont été abordés, notamment les représentations de l'enfermement carcéral, les conditions de détention, l'utilisation du temps de détention, les interactions avec divers groupes d'acteurs à l'intérieur et à l'extérieur de la prison, et les conséquences de l'incarcération sur la sphère familiale, le travail, l'éducation et la santé. Pour contraster les trajectoires de vie, nous avons diversifié notre échantillon en fonction de critères tels que l'origine 
ethnoculturelle, l'âge, la scolarité, la durée de la sentence et le nombre d'incarcérations ${ }^{3}$.

\section{Conditions de vie actuelles des répondantes autochtones}

Parce que les conditions de vie générales des répondantes au moment des entrevues constituent inévitablement le «point d'arrivée» des trajectoires de vie que nous avons analysées, il convient d'en présenter, ici, les pourtours les plus intéressants. Au moment des entrevues, trois répondantes sont incarcérées, mais résident dans leur communauté d'origine au moment de leur incarcération, alors que les quatre autres n'ont pas, au moment des entrevues, de démêlé avec la justice et vivent à Montréal, en milieu urbain. De ces sept répondantes, deux vivent en union libre alors que les autres sont célibataires, et aucune d'entre elles n'a d'enfant à sa charge au moment des entrevues. Toutes les participantes vivent de prestations d'aide sociale, à l'exception d'une répondante qui vit des revenus de la quête. Deux répondantes ont cessé la prostitution depuis quelque temps en raison d'une détérioration de leurs conditions de santé ou de la non-acceptation de cette pratique par leurs nouveaux conjoints. Alors que quatre femmes autochtones vivent en logement, trois sont sans domicile fixe. Par ailleurs, trois répondantes affirment avoir cessé la consommation de substances psychotropes quelques mois avant la tenue de nos entrevues. À l'exception de deux répondantes qui affirment se trouver en bonne santé, les conditions de santé des autres femmes sont fragilisées alors que trois d'entre elles sont atteintes du VIH-Sida, une souffre d'un cancer, deux autres ont des dysfonctionnements hépatiques, certaines font de l'anémie, plusieurs ont des problèmes pulmonaires et, enfin, d'autres souffrent de diabète sévère. Ces conditions témoignent d'une certaine homogénéité parmi les répondantes. L'ensemble de ces conditions dénote, en effet, la fragilité et la marginalisation des répondantes dans des filières où les institutions de prise en charge (d'aide et de contrôle) occupent une place importante.

Ce bref aperçu des conditions de vie générales des participantes, telles qu'elles ont été saisies au moment des entrevues, cherche à jeter

3. La dernière expérience d'incarcération ne remontant pas à plus de trois ans afin de mieux homogénéiser le contexte carcéral dans lequel les femmes autochtones ont pu évoluer. 
une lumière sur ce que nous avons convenu d'appeler le «point d'arrivée» des trajectoires de vie des femmes autochtones. Or, ce point d'arrivée ne peut prendre sens que s'il est mis en rapport avec le «point de départ» de ces mêmes trajectoires de vie, ce que nous circonscrivons comme étant les conditions du milieu d'origine dans lequel les participantes ont évolué au cours des premières années de leur existence.

\section{Les conditions du milieu d'origine des femmes autochtones}

Ces conditions du milieu d'origine correspondent aux conditions de vie familiale dans lesquelles les femmes autochtones évoluent au cours de leurs premières années de vie. Bien que l'ensemble des participantes à notre étude ait connu des conditions de vie ardues dans leur milieu d'origine, il est possible de distinguer deux groupes: un premier qui regroupe des femmes ayant bénéficié de conditions de vie familiale plus stables (trois répondantes: Mary, Lucy et Sarah) et un second qui réunit des femmes ayant connu des conditions nettement plus précaires et instables (quatre répondantes: Paula, Laura, Emma et Carry). Le premier groupe peut être qualifié de plus stable dans la mesure où les femmes le composant ont grandi auprès de leurs parents naturels, dans une famille dont les deux parents occupaient des emplois réguliers et rémunérés ${ }^{4}$ (charpentier, fonctionnaire, aide aux bénéficiaires). Bien que la violence familiale et conjugale soit présente au sein du milieu familial de ce groupe de femmes, celle-ci se manifeste de façon sporadique. Par ailleurs, aucune participante ne fait état de problèmes de consommation abusive de substances psychotropes chez leurs parents, ni durant leur enfance ni au cours de leur adolescence. Le discours de Sarah témoigne du climat familial relativement stable dont elle a pu bénéficier au cours de son enfance:

Nous autres on allait dans le bois, tout l'été on restait là, on ramassait des bleuets pis il fallait aller camper là [...] on vivait un peu de ça là pis après ça ben ça buvait pas ben ben $[\ldots]$ dans ma famille ça buvait là, mais pas comme moi je bois là maintenant, avant je ne buvais pas mais asteur je bois en masse là [...] (rires), eux-autres c'était plus rare, juste une fois de temps en temps vraiment $[\ldots]$.

4. Une seule participante évolue dans une famille dont le revenu est assumé par un seul parent. 
Le milieu familial du second groupe de femmes paraît davantage problématique et instable. Trois des quatre répondantes de ce groupe n'ont pas connu leurs parents naturels, ayant été adoptées dès leur naissance par des membres de la famille élargie ou par des amis de la famille. Pour certaines, comme Carry, l'adoption en bas âge résulte d'une grossesse hâtive chez sa mère biologique. Ainsi, elle raconte:

I was adopted only two days after when I was born, to get away [be]cause my pregnant mother was too young, she was only 14 or 15, sometbing like that. She didn't want me [be] cause she wanted to be free and play around. So, I lived with my parents only for 2 days, after that I was adopted by a couple, some friends of my parents.

La situation socio-économique des parents naturels de même que celle des parents adoptants est plus précaire que celle des parents du premier groupe de femmes. En effet, dans tous les cas, le revenu familial est assumé uniquement par le père (la mère demeurant au foyer), lequel occupe généralement des fonctions de manœuvre (hôtel, foresterie, commis-vendeur). Toutes les femmes de ce groupe rapportent qu'au moins un des deux parents (naturels ou adoptants) a des problèmes de surconsommation d'alcool et/ou de drogues. Par ailleurs, les quatre femmes de ce groupe affirment avoir été témoins de violence conjugale entre les parents ou, encore, avoir été victimes de violence de la part de leurs parents ou de leur fratrie. Emma a été victime de violence de la part de ses frères: «[...] ils me battaient au bout mes frères, ah oui ils me crissaient des coups de pieds partout des fois là, ils étaient sur le PCP [hallucinogène stimulant] pis j'en mangeais toute une, je te jure, ça a été l'enfer quand j'étais jeune, j'ai des cicatrices encore de ça.»

La comparaison des conditions de vie des répondantes autochtones en leur «point de départ», ainsi qu'en leur "point d'arrivée» montre que les différences observées chez les deux groupes de femmes en amont - c'est-à-dire au «point de départ» des trajectoires de vie - s'estompent à l'arrivée. Comme l'illustre de façon schématique la figure 1 ci-dessous, les femmes autochtones du second groupe sont maintenues dans des conditions de vie fragilisées et précaires, alors que celles du premier groupe connaissent une nette détérioration de leurs conditions de vie au cours de leur trajectoire.

Bien que la figure 1 schématise le mouvement général des conditions de vie des femmes autochtones de manière sommaire, elle ne permet pas d'identifier la présence de ruptures pouvant conduire à des formes 
F I G URE 1

Mouvement général des conditions de vie des répondantes

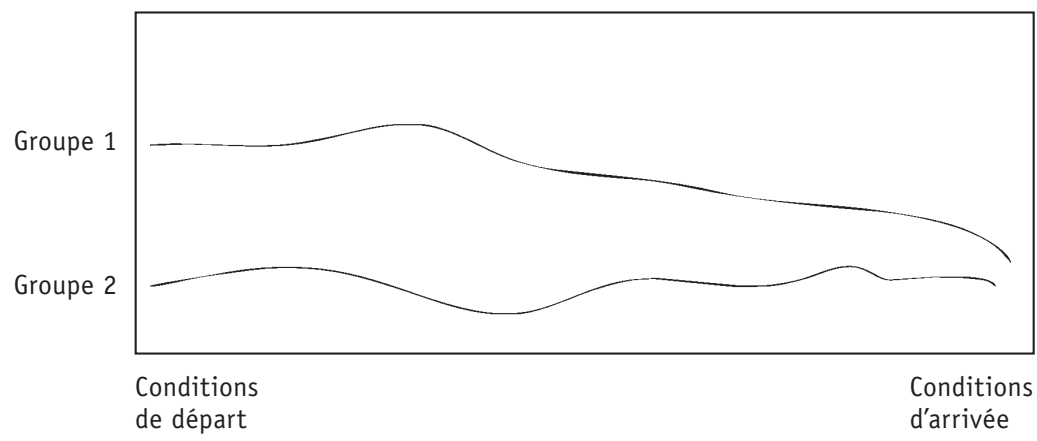

d'exclusion sociale au cours des trajectoires de vie des participantes. À cet effet, les sections ultérieures de cet article tentent de mettre en exergue les principales discontinuités vécues par les femmes au sein d'espaces d'insertion sociale tout au long de leurs trajectoires de vie.

\section{Des conditions de départ aux premières judiciarisations}

Nos analyses montrent que les femmes des deux groupes connaissent un enchaînement plus ou moins successif de ruptures avec les espaces d'intégration (ex.: famille, travail, éducation), lesquelles permettent de comprendre de quelle manière et dans quelles conditions elles en viennent à entrer en contact avec le système de justice pénale et, ultimement, avec l'institution d'enfermement carcéral. Tout d'abord, toutes les répondantes connaîtront des ruptures avec leur milieu familial au cours de leur vie. Alors que les fugues et les mauvais traitements infligés par les parents constituent des facteurs de séparation temporaire communs aux deux groupes de répondantes, la principale différence entre les femmes qui ont évolué dans un milieu familial plus stable et celles qui proviennent de milieux de vie très problématiques réside dans la précocité et l'intensité de ces ruptures ainsi que dans la nature de la prise en charge qui en découle. Les femmes autochtones du premier groupe vont vivre des ruptures temporaires avec le milieu familial dans le sens où elles connaitront des placements de courte durée au sein des membres de la famille élargie. Bien que séparées de leurs familles d’origine, 
ces répondantes peuvent néanmoins conserver un minimum de liens avec elles. Le discours de Lucy témoigne de ces courtes ruptures: «I didn't like my stepmother, so I ran away a while to my relatives, you know. I've got big family up there, I did that often. I called my father sometimes but I ran away to different aunts and cousins, you know. I stayed there for a while. [...]» Certaines femmes du second groupe seront abandonnées dès leur naissance en raison d'un rejet de leur mère ou des ressources économiques insuffisantes de leurs parents alors que d'autres connaîtront l'abandon plus tardivement à l'occasion d'une séparation de leurs parents. À cet effet, Paula raconte: «Suddenly, she went out and moved with this man and she didn't tell anybody. She let us in the house alone, without control, we were all the children alone.» L'instabilité familiale que les femmes de ce groupe connaissent très jeunes suscite l'intervention d'institutions de protection sociale ayant pour conséquence le placement institutionnel pour de longues périodes dans des familles ou centres d'accueil ou encore dans des pensionnats. Les constants mouvements de va-et-vient entre les différents lieux de placement ont toutefois eu pour conséquence d'accroître le caractère fragile et conflictuel des liens familiaux. Ces conditions rendent les femmes de ce groupe beaucoup plus à risque d'adopter des comportements problématiques susceptibles d'entraîner l'intervention de la police. Ces premiers gestes délictuels et la judiciarisation qui s'ensuit renforceront alors les ruptures avec le milieu familial.

Comme on le constate, les conséquences des problématiques familiales et des actes commis par les répondantes diffèrent dans les deux groupes: le groupe de répondantes ayant eu un contexte familial plus stable dispose de ressources informelles de prise en charge directement issues de leur réseau relationnel qui intervient uniquement dans un contexte de protection et non de délinquance. Pour sa part, le groupe de répondantes dont les conditions de départ sont davantage précaires est régulièrement pris en charge par des structures formelles d'intervention, et ce, autant dans les cas de délinquance que dans les cas de protection.

Bien que distinctes, l'intensité, la fréquence et la précocité des ruptures et des interventions institutionnelles auront des répercussions similaires sur l'insertion dans l'espace éducationnel pour les deux groupes de répondantes. À titre d'exemple, toutes les femmes de notre étude ont abandonné le milieu scolaire au cours de leurs études secondaires et les motifs d'abandon invoqués par les répondantes ne permettent pas de discriminer entre les deux groupes. Certaines de ces ruptures scolaires 
surviennent à la suite d'impondérables qui exhortent les femmes autochtones à interrompre de manière définitive leur parcours scolaire. C'est le cas de deux répondantes pour qui l'arrivée d'une grossesse non planifiée et le départ précipité de la mère les poussent à devoir assumer le rôle de mère au foyer ${ }^{5}$. La perception négative de l'école et des acteurs qui y sont présents conduit certaines autres répondantes à fréquenter de manière plutôt irrégulière le milieu scolaire. Le cas de Sarah illustre ce désintérêt fréquent à l'égard de l'institution scolaire: «Je n'aimais pas l'école moi, je n'aimais pas les maîtresses pis je n'étais pas bien à l'école. J'y allais pas souvent, je me sauvais de là avec mes amis pour aller jouer.» Outre cette forme d'indolence vis-à-vis de l'école, d'autres motifs d'abandon scolaire sont davantage reliés aux conditions et au style de vie des femmes autochtones tels que la surconsommation de substances psychotropes, les mauvais traitements de la part des parents ou encore la violence conjugale des parents qui poussent certaines répondantes à demeurer à la maison, notamment dans le but de protéger la mère violentée.

Les conditions de vie dans lesquelles tendent à évoluer les répondantes autochtones semblent inquiétantes au point où elles incitent parfois les acteurs de l'institution scolaire à les dénoncer aux institutions de prise en charge. Bien que ces signalements soient des décisions normatives qui visent à protéger le développement de l'enfance et de l'adolescence, de telles décisions entraînent, par la même occasion, l'intervention d'institutions de prise en charge formelle qui viennent participer et renforcer les ruptures familiales et éducationnelles, tel que l'explique Emma :

Je manquais beaucoup l'école. Un moment donné, le directeur a téléphoné aux services sociaux pis ils ont communiqué avec mes parents. Il leur a dit: «ben votre fille est jamais à l'école, pis c'est quoi qui se passe, pis on va être obligé de la placer». Ils m'ont placée pour un mois dans une autre ville.

Pour leur part, les signalements non effectués par l'institution scolaire provoquent, eux aussi, des discontinuités se traduisant par de fréquents changements d'établissement scolaire. Ce faisant, les femmes peinent à s'intégrer pleinement à la sphère scolaire et leur parcours au sein de

5. Soulignons que cette situation n'est pas perçue, par les répondantes, comme une barrière à la poursuite du cheminement scolaire, mais plutôt comme une occasion d'apprendre à accomplir les tâches familiales et à assumer le rôle de mère au foyer. 
cet espace d'intégration sociale se retrouve ponctué de fréquentes interruptions.

En fin de compte, si les conditions de départ du milieu familial, ainsi que les discontinuités dans les liens familiaux étaient notablement différentes entre les deux groupes de répondantes, ces distinctions s'estompent lorsque l'on examine les liens que les répondantes entretiennent avec l'institution scolaire. La récurrence des absences scolaires conduira, en dernière instance, toutes les répondantes à abandonner définitivement la fréquentation de l'établissement scolaire. Dans notre corpus, aucune femme autochtone n'est parvenue à gravir les échelons scolaires au-delà de la troisième année d'éducation secondaire. Or, la rupture définitive avec l'espace scolaire contraint les femmes autochtones à trouver des sources de revenu qui leur permettent de subvenir à leurs besoins. Ici, des distinctions très nettes apparaissent entre les deux groupes de répondantes. Tandis que les femmes ayant bénéficié de conditions de départ plus stables ont tenté quelques expériences d'emploi régulier, les femmes du deuxième groupe (à l'exception d'une répondante) n'auraient entrepris aucune démarche formelle d'insertion en emploi. Par ailleurs, en raison de leur sous-scolarisation, les femmes qui tentent d'accéder à des emplois réguliers décrochent surtout des occupations contractuelles de courte durée et peu rémunérées telles que gardienne d'enfants, sondeuse, caissière, plongeuse dans un restaurant ou, encore, préposée dans un hôtel. Cette instabilité dans l'insertion en emploi sera, pour le reste, consolidée par des conditions de vie qui viendront précipiter l'exclusion définitive des femmes autochtones de cette sphère d'activité sociale. À l'exception de la violence conjugale - qui apparaîtra plus tard dans leur parcours -, les conditions de vie ayant contribué à miner les liens familiaux et à forcer les discontinuités scolaires sont les mêmes que celles qui précipiteront l'abandon du marché de l'emploi, à savoir la surconsommation de substances psychotropes principalement.

Les femmes autochtones qui sont issues d'un milieu familial plus stable, et qui sont également les moins institutionnalisées, tendent à demeurer en communauté et à dépendre de différentes institutions sociales d'aide (aide sociale, maison d'hébergement, centre de désintoxication). De plus, n'ayant pas eu de contact formel avec le système de justice pénale pour adolescents, c'est plutôt à l'âge adulte que ces femmes connaîtront leurs premiers démêlés avec l'appareil pénal. À titre d'exemple, certaines femmes qui vivent dans un contexte de violence 
conjugale sont amenées à commettre des actes de violence à l'endroit de leur conjoint agresseur. C'est précisément dans un tel contexte que deux des trois répondantes de ce groupe ont dû purger des peines d'incarcération pour voies de fait ${ }^{6}$.

En ce qui concerne les répondantes provenant de milieux plus désaffiliés - et n'ayant jamais tenté l'insertion en emploi -, elles dépendent essentiellement des prestations de l'aide sociale ou s'intègrent dans des réseaux de prostitution et de danse érotique pour subvenir à leurs besoins. À l'opposé des femmes qui composent le premier groupe, les femmes du second groupe tendent à quitter la communauté autochtone d'origine pour les grands centres urbains. Là, elles s'enracinent peu à peu au sein de différents espaces de vie où elles rencontreront des acteurs qui vivent les mêmes réalités qu'elles (les débits de boisson, la rue, le milieu de la drogue, de la prostitution et de la danse). Ces acteurs constitueront peu à peu leur principal, voire leur unique réseau relationnel. L'insertion au sein de ces espaces «à risques» et, encore plus, le style de vie qui y est associé conduiront les répondantes de ce groupe à vivre de la violence conjugale, à commettre des actes de violence ou, encore, à dépendre de sources de revenus illicites (ex.: fraude, vente de drogues, prostitution, danse érotique). La visibilité urbaine de telles conditions de vie rend alors ces femmes davantage vulnérables à la vigilance policière. Ce regard, rappelons-le, est familier pour ce groupe qui a déjà été assujetti à de fréquents placements en institution tout au long de l'adolescence. Une telle visibilité au regard des agences de contrôle social décuple, dès lors, les risques de voir sa trajectoire de vie ponctuée de séjours en détention.

\section{L'expérience de l'enfermement carcéral ${ }^{7}$}

Pour le premier groupe de femmes autochtones peu institutionnalisées, l'expérience de l'incarcération se produit relativement tard à l'âge adulte et les peines purgées sont habituellement de courte durée. Cependant, alors que l'emploi, l'éducation et les programmes correctionnels sont des composantes estimées être décisives pour l'insertion sociale des hommes et des femmes détenus, ces programmes n'étaient pas accessi-

6. La troisième répondante a été incarcérée pour des infractions liées à la consommation d'alcool dans des lieux publics.

7. Nous résumons ici les principales analyses d'un article antérieur portant sur les expériences d'enfermement des femmes autochtones (Brassard, 2006). 
bles, au moment de nos entrevues, aux femmes autochtones assujetties à de courtes sentences au Québec. Cela a pour effet de réduire considérablement les possibilités de développer certaines habiletés chez les répondantes. Cette réalité met en lumière l'un des paradoxes de l'institution carcérale qui, en limitant l'accès à des programmes correctionnels qui favorisent l'insertion sociale, participe au maintien de la non-intégration des détenus au sein de sphères déterminantes de la vie sociale.

Sur le plan des relations sociales, soulignons que ce ne sont pas toutes les femmes autochtones qui ont la possibilité de se prévaloir de leurs droits de visite en prison. Pour les femmes autochtones du premier groupe, l'enfermement carcéral a pour conséquence de provoquer une rupture temporaire avec le réseau relationnel puisque leur famille et leurs enfants vivent en communauté éloignée et ne peuvent payer les coûts élevés du transport vers la prison. Ainsi, les seuls visiteurs sur lesquels peuvent compter ces femmes incarcérées sont les conseillers parajudiciaires autochtones du Québec. Ces conseillers effectuent des visites en prison afin de répondre aux besoins des femmes et de s'assurer qu'elles sont adéquatement informées des services, des programmes et des ressources pouvant leur venir en aide en cours d'incarcération ou à leur sortie. À l'exception d'une seule détenue amérindienne qui affirme n'avoir point besoin de tels services, toutes les autres participantes de ce groupe affirment connaître les conseillers autochtones et avoir utilisé leurs services en détention. Certaines femmes, comme Sarah, savent qu'elles peuvent compter sur un personnel apparemment qualifié pour rendre des services de toutes sortes:

Ils te donnent beaucoup de services, ça donne un bon coup de main, surtout quand tu es en prison. Ils s'en vont voir si tu es bien pis tout [...]. Au moins, avec eux autres, tu sais tu es en prison, eux autres ils s'en vont te rendre visite pour savoir si tu vas bien, si tu veux changer d'avocat, si ton avocat est correct ou ben, tu sais, t'as besoin de quoi [...]. C'est très commode le parajudiciaire.

Pour les femmes dont le parcours de vie témoigne d'une institutionnalisation récurrente - celles issues du second groupe -, la prison ne constitue qu'un espace institutionnel parmi d'autres dans une trajectoire marquée dès l'adolescence par de multiples placements institutionnels. L'incarcération s'insère dans un mouvement, une continuité et une logique de prise en charge institutionnelle déjà intégrée au style de vie des femmes qui appartiennent au second groupe. Résignées à ce que l'institution carcérale intervienne à un ou plusieurs moments de leur 
vie, ces femmes en viennent à utiliser la prison pour répondre à certains besoins. Vivant couramment dans des conditions de grande précarité (itinérance, prostitution, surconsommation de drogues, violence, etc.), les femmes autochtones de ce groupe perçoivent la prison non pas comme une institution à caractère fondamentalement punitif, mais comme un espace où elles peuvent prendre un moment de pause, une occasion de se refaire une santé ou, encore, un lieu d'ancrage et d'inscription résidentielle que le milieu de la drogue, de la danse érotique et de la rue ne permet pas. Laura exprime son rapport à l'enfermement carcéral et l'impact que ce passage a provoqué sur sa santé physique:

J'appelais mon avocat, je l'appelais pis j'y disais: «Je veux sortir, je suis assez reposée, là, j’ai engraissé, là, pis là je suis ben correcte. Fais-moi sortir de là [...].»C'est comme si j'allais me reposer. C'est comme si c'était un genre de, de chambre [...] jusqu'à tant que je prépare mon autre coup. Pis là il disait: «OK, mais si tu me promets de ne plus rien faire, là.»

Contrairement aux femmes du premier groupe, les femmes autochtones qui composent le second groupe purgent de plus longues peines et la majorité d'entre elles participent activement aux différents programmes correctionnels et services offerts en milieu carcéral. Cependant, les motivations qui incitent les femmes de ce groupe à participer aux programmes correctionnels naissent moins d'une volonté intrinsèque d'acquérir des habiletés ou d'entamer un cheminement personnel que d'une stratégie visant essentiellement à écouler le temps de détention et à éviter l'isolement social rattaché à la prise en charge carcérale. Par ailleurs, en prison, les femmes autochtones doivent composer avec l'image de «détenues redoutables qu'il faut craindre» que les détenues non autochtones entretiennent souvent à leur égard. Cette image concerne plus particulièrement les femmes amérindiennes davantage institutionnalisées, celles qui sont régulièrement perçues comme des détenues violentes et alcooliques, ne reculant devant rien. Ces répondantes tendent à être plus fréquemment impliquées dans des incidents de violence au sein desquels elles tendent également à se servir des stéréotypes qui leur sont accolés par les détenues non autochtones. Laura raconte: «j'ai fait chanter une fille, j'ai dit si tu ne fais pas ce que je te demande on va te crisser une volée là. Pis moi je suis indienne là, pis les Indiennes là quand elles font de quoi [...]. Pis là la fille est allée le faire tout de suite là, elle avait trop peur des Indiennes (rires).» La promiscuité qu'impose la prison aux personnes détenues issues de 
conditions de vie similaires permet de reproduire, chez les femmes autochtones, des filières d'acteurs foncièrement identiques à celles qui prévalaient dans leur vie avant leur incarcération (prostitution, réseau relationnel de vente de drogues), des filières que les femmes retrouveront, d'ailleurs, en société au terme de leur incarcération. À cet effet, Emma mentionne que lorsqu'elle est détenue, elle croise fréquemment, en prison, les mêmes personnes qu'elle côtoyait à l'extérieur des murs de la prison: «Ben ça faisait pas trop de changement parce que en dedans [en prison], c'était les mêmes filles avec qui je vendais dehors. Faque, moi j'aimais ça parce qu'on se retrouvait, on avait du fun.»

\section{Les effets de l'incarcération dans la trajectoire post-carcérale ${ }^{8}$}

Les recherches qui se sont intéressées aux effets de l'enfermement carcéral dans la trajectoire de vie de populations exclues et marginalisées ne sont pas nombreuses au Québec (Landreville et al., 1981; Pires, 1983; Hamelin, 1989; Laberge et Morin, 1997; Landreville, 2002). Nous avons recensé moins d'une dizaine d'études portant expressément sur les effets de l'incarcération dans la vie d'individus vulnérables et la grande majorité de ces dernières a surtout mis l'accent sur le rôle et les effets négatifs de l'incarcération. Seule l'étude de Landreville et al. (1981) a soulevé que l'impact du passage au sein du système carcéral pouvait être différentiel. À partir de trois groupes de répondants issus de classes sociales différentes, Landreville et al. (1981) ont notamment observé que la classe sociale avait un impact déterminant sur les mécanismes de production et de neutralisation des coûts sociaux ${ }^{9}$ ainsi que sur la distribution des biens négatifs de l'appareil pénal. Cette ouverture analytique est particulièrement pertinente pour notre étude.

En effet, nous avons déjà noté que les femmes autochtones les moins institutionnalisées résident le plus souvent en communauté autochtone. Vivant pour la plupart en logement et maintenant des contacts fréquents

8. Dans les cas où les femmes autochtones avaient connu plusieurs expériences d'enfermement carcéral, nous avons privilégié, dans la mesure du possible, une analyse des effets de chaque expérience carcérale afin de distinguer des effets différenciés selon la peine d'incarcération.

9. Les coûts sociaux sont définis comme: «Toutes les pertes, désavantages, atteintes, conséquences négatives subis ou portés par une classe, une fraction de classe, un groupe ou un être humain en raison de l'existence ou du fonctionnement du système pénal» (Landreville et al., 1981: 35). 
avec leurs familles, les femmes autochtones de ce groupe sont amenées à rompre avec leurs familles, leur réseau relationnel et à quitter leur domicile pour purger leur peine d'incarcération, souvent à plusieurs centaines de kilomètres de leur communauté d'origine: "I have my house back home, you know. I leaved [sic] everything up there, I'm bere only for do [sic] my time in Tanguay. I have everything. My family takes care of my things up North» (Mary). Chez les femmes autochtones du second groupe - celles qui vivent en milieu urbain et fortement institutionnalisées -, l'incarcération introduit des ruptures différentes, notamment une rupture provisoire avec des conditions et des espaces de vie au sein desquels l'exposition prolongée devenait insupportable (que l'on pense, par exemple, à la rue ou au milieu de la prostitution). L'incarcération peut représenter, dans ces cas, une occasion de remettre de l'ordre dans sa vie. De l'avis de certaines femmes autochtones institutionnalisées, l'incarcération constituerait, dans les faits, un moment qui vient neutraliser l'effet de certaines conditions de vie fragilisées. Comme le mentionne Chantraine (2004 : 57-58), la prison constituerait, alors, la seule structure étant en mesure de structurer ces individus. Bref, au sein de cette institution, certaines femmes se sentent en contrôle d'elles-mêmes: «Every time when I go out from jail, I would drink or party and get myself in trouble. Jail was a place where I felt secure, more structure» (Paula).

De façon générale, les premières expériences d'incarcération laissent des traces indélébiles dans la biographie des femmes autochtones. À cet effet, un des stigmates avec lesquels les répondantes autochtones doivent composer au quotidien est le casier judiciaire. Déjà en 1969, le rapport du juge Ouimet, alors président du Comité canadien de la réforme pénale et correctionnelle, avait vertement dénoncé les effets importants des antécédents judiciaires sur le processus de réintégration sociale des contrevenants (Ouimet, 1969). Depuis, maintes études étatsuniennes, européennes et canadiennes (Hattem et Parent, 1982; Pires, 1983; Hamelin, 1989; Laberge et al., 1998), ont abondamment confirmé que les conséquences légales de condamnations pénales sont non seulement nombreuses, mais que l'un des effets les plus décourageants du casier judiciaire est sans conteste la difficulté de se trouver un emploi. Le stigmate et l'opprobre social qui sont associés au casier judiciaire rendent ainsi les personnes criminalisées, et particulièrement celles qui ont été incarcérées, discréditables. L'analyse des trajectoires de vie des répondantes autochtones prend une direction similaire à celle déjà tracée dans la littérature existante. Nos analyses suggèrent, en effet, que, après 
l'incarcération, les femmes autochtones des deux groupes qui tentent de s'insérer dans différents espaces d'intégration, tel que l'emploi, s'en voient généralement refuser l'accès par les employeurs en raison de leur casier judiciaire. Ici, l'expérience de Laura dresse un portrait représentatif des obstacles auxquels se butent les femmes autochtones judiciarisées et le découragement qui s'ensuit. N'ayant connu aucune expérience de travail au cours de sa vie, sauf à l'intérieur de la prison, Laura entreprend des démarches d'accès à l'emploi quelques mois après sa libération. Après quelques tentatives, elle se rend compte que l'accès au marché du travail est absolument bloqué et que cet espace ne constitue pas une voie d'intégration réaliste pour elle. Elle décide donc de rejoindre les réseaux clandestins de la danse érotique et de la prostitution pour assurer sa subsistance économique:

Au début, j'ai essayé de m'engager dans un motel. Quand ils ont vu que j'avais un dossier, ils m'ont dit qu'il y avait une autre femme qui était déjà acceptée. Je me suis dit: «à cause de mon dossier je ne serai jamais capable de travailler», mais je ne peux pas vivre sur le Bien-être, ce n'est pas assez, la seule chose que je pouvais faire c'est de continuer à danser, pis j'avais la gaffe [prostitution] aussi là.

Même si certaines autres répondantes autochtones ont connu des expériences d'insertion en emploi dans le passé, leur casier judiciaire opère une rupture définitive avec cet espace. Ainsi, les répondantes possédant un casier judiciaire sont acculées à un dilemme de taille: avouer à un employeur potentiel l'existence dudit casier et risquer d'être éliminée du processus d'embauche, ou taire son existence et risquer d'être congédiée au moment où leurs fausses déclarations seront mises au jour. La réinsertion sociale par l'entremise de l'emploi devient alors impossible pour ces femmes, et ce, même dans leur communauté d'origine, comme Lucy l'a malheureusement constaté:

I applied up North for a childhood protection and they said: «Ob no!» My dad was working there and so be asked me one time: " that's why you cannot work here». If he wasn't my dad, I would not know about this, you know. They knew I bad a [criminal] record through the computer. I didn't find a job because I bave record in Tanguay. Over here, it travelled, they saw, so I couldn't find a job so I got frustrated more.

Dans la foulée du dilemme que pose, pour la personne judiciarisée, l'existence d'un casier judiciaire, nos analyses montrent qu'un dilemme similaire se forge aussi au moment de la recherche d'un logement. Si le 
casier judiciaire ne semble poser aucun problème insurmontable pour les femmes autochtones qui vivaient déjà en logement avant leur incarcération, il en est autrement pour les répondantes qui n'avaient pas d'adresse résidentielle formelle avant leur incarcération. Après avoir essuyé quelques refus de la part de propriétaires, certaines répondantes usent de différentes stratégies afin de contourner le stigmate du casier judiciaire et accéder au logement. Elles vont alors utiliser le nom d'un conjoint ou d'une connaissance sans casier judiciaire ou, encore, tenter de négocier la location du logement avec le concierge au lieu du propriétaire:

Je m'arrangeais pour que le loyer ne soit jamais à mon nom. Je savais qu'ils pouvaient me refuser aussi pour le logement. Ou je pognais toujours des concierges. Au moins les concierges, avec eux autres t'as pas de problème quand t'as un dossier [judiciaire].

À l'instar des études portant sur les coûts sociaux de l'appareil de justice pénale, nos analyses mettent également en lumière l'existence de pratiques sociales de sélection systématique discriminatoire sur la base du casier judiciaire (Landreville, 2004). Pour les femmes autochtones interviewées, cette sélection traduit la perpétuation de la stigmatisation spécifique que produit le passage en prison. Ainsi, au terme de la peine purgée, la scission du corps social que symbolisait à l'origine la peine d'exclusion carcérale (Combessie, 2004) semble, elle aussi, subsister de sorte que la femme «reprise de justice» demeure toujours, malgré son retour en communauté, une «reprise de justice».

\section{Les effets des règles institutionnelles sur le parcours de vie post-carcéral}

Les prisons provinciales canadiennes offrent une gamme limitée, voire inexistante, de programmes de réinsertion aux détenus (es) en raison de trop brefs séjours en détention prévus. Un tel désœuvrement vient ainsi annihiler l'objectif manifeste que se donne l'institution carcérale, à savoir l'amendement et la réforme des détenus que l'on isole à la fois spatialement et temporellement. À cet égard, Lucy explique le faible impact qu'aurait eu son expérience d'incarcération sur sa surconsommation d'alcool:

Jail didn't really bave an impact. I continued to drink after as usual. I love my alcobol, that's my problem [...]. It doesn't change notbing. I don't know, for some reason [prison] doesn't seem to bave an impact on me. 
À l'exception d'une seule répondante, qui affirme que ses premières incarcérations avaient contribué à améliorer et consolider les liens familiaux qu'elle croyait rompus, l'incarcération ne vient influencer ni les relations conflictuelles familiales ni les ruptures relationnelles, car celles-ci sont davantage conditionnées par un contexte de vie qui prévalait déjà dans la vie de plusieurs femmes autochtones avant leur incarcération (distance avec la communauté d'origine, relations conflictuelles avec la famille, perte de la garde légale des enfants). En réalité, la détention maintient, mais ne crée pas cet état de fait.

L'incarcération renforce également le sentiment de révolte et d'amertume que les répondantes fortement institutionnalisées entretiennent déjà à l'égard de leur vie (Ross, 1998; Grobsmith, 1995). Pour ces femmes, la période post-carcérale leur fait prendre conscience que rien n'a changé dans leur vie. Étant toujours en rupture avec les principaux espaces d'insertion, ces répondantes ne parviennent pas davantage, après leur libération, à se rattacher aux espaces d'insertion normalisés et à espérer une vie meilleure. Certaines se résignent et retournent vers les milieux de vie où elles sont déjà fortement insérées comme le milieu de la prostitution, de la drogue ou de la danse érotique:

Quand je sortais, je n'avais plus rien là, j'étais dans la rue comme avant. J'ai recommencé à me piquer pis à faire la gaffe, ma vie était complètement finie, faque y avait plus rien pour me retenir.

Faisant l'objet de répression policière récurrente en milieu urbain, certaines femmes autochtones constatent que ce milieu n'offre pas l'invisibilité et l'anonymat qui permettent d'éclipser un tant soit peu le stigmate pénal et le sceau de l'infamie que porte la justiciable. Certaines décident alors de retourner en communauté autochtone dans l'espoir de neutraliser les effets de leur parcours judiciaire. De retour dans leur communauté d'origine, elles constatent, toutefois, que celle-ci ne constitue pas davantage un territoire exempt de stigmatisation. S'agissant la plupart du temps de communautés autochtones exiguës, leurs membres sont rapidement mis au courant de l'emprisonnement des répondantes. Certaines, comme Emma, vivent un fort sentiment de rejet et d'exclusion de la part des leurs et n'hésitent pas, en ultime recours, à retourner en milieu urbain dans le but d'échapper à cette pression sociale qu'elles jugent humiliante:

Les gens savaient en communauté que j'avais fait de la prison, ils me regardent pis ça placotte. Je ne me sens pas aimée comme avant. Mais c'est 
surtout mes enfants, ils ont vécu une histoire très triste parce qu'à l'école les autres enfants [...] disaient: «Ta mère est toujours en prison.» Mes enfants ont vécu du rejet à l'école. Je suis revenue icitte [en milieu urbain] parce que j'étais pus capable d'endurer ça.

Prises au cœur d'un enchevêtrement de logiques ségrégatives, celles de la prison bien sûr, mais également celles de la communauté des «honnêtes gens", les femmes autochtones dont les trajectoires de vie sont ponctuées de passages en prison peuvent se concevoir, à l'instar de ce qu'on trouve dans les travaux de Girard (1972), comme des justiciables sacrifiées, c'est-à-dire des boucs émissaires constituant, pour les membres de communautés humaines - par ailleurs séparés par des intérêts divergents -, un de leurs multiples exutoires communs.

\section{Conclusion}

L'utilisation des trajectoires comme outil de reconstruction des parcours de vie a permis de constater que les distinctions observées dans les conditions de départ (milieu d'origine) s'estompent puisque les conditions de vie au point d'arrivée des trajectoires sont relativement similaires entre les deux groupes de femmes autochtones. Cette observation a suscité une question importante: quel est le poids du processus pénal 1) dans la détérioration notable des conditions de vie du groupe de répondantes les plus stables dans leur milieu d'origine, et 2) dans le maintien de conditions de vie déjà fragilisées chez les répondantes issues des milieux plus problématiques? La distinction observée entre les conditions de vie de ces deux groupes de répondantes se maintient pratiquement jusqu'aux expériences de détention (à l'exception, toutefois, du rapport que les femmes autochtones entretiennent, mais surtout n'entretiennent pas, avec l'espace scolaire et avec le marché du travail). Or, même si le groupe issu d'un milieu plus stable procède à des tentatives d'insertion en emploi, ces tentatives tendent à échouer et les deux groupes finiront par délaisser définitivement cette sphère d'activité. La sous-scolarisation joue, ici, un rôle important dans la désinsertion en emploi. Parallèlement, cette désinsertion contribue à maintenir les répondantes dans des filières de prise en charge ou de modes de subsistance qui les rendent plus susceptibles d'entrer en contact avec les instances de contrôle socio-pénal. Ainsi, la détérioration des conditions de vie des répondantes les moins vulnérables au point de départ est 
certainement conditionnée par la double désinsertion qui s'opère sur le plan de la sphère scolaire et de celle de l'emploi.

S'il est possible de repérer des causes de détérioration et de maintien des conditions des deux groupes de répondantes prévalant avant l'expérience carcérale proprement dite, les effets les plus directs du passage dans l'institution d'enfermement sont produits par le stigmate du casier judiciaire et par les règles d'accessibilité aux programmes correctionnels offerts en prison. Paradoxalement, le groupe le moins vulnérable est celui qui a le moins accès aux programmes en raison de la courte durée des peines. Le groupe le plus vulnérable, par contre, a accès aux programmes correctionnels, mais les visées des femmes à cet égard ne s'inscrivent pas dans la logique du système carcéral. En effet, au lieu de s'engager dans des programmes de réinsertion pour «corriger» certaines problématiques de leur vie, les répondantes autochtones entreprennent des programmes correctionnels dans un but davantage instrumental: écouler leur temps de détention et éviter l'isolement fréquemment réservé aux détenues qui refusent de participer.

Le casier judiciaire contribue à exclure définitivement les répondantes des deux groupes du marché de l'emploi. Il vient également décupler les difficultés liées à l'accès au logement et provoque la marginalisation des femmes dans leur communauté d'origine. Aussi, le casier judiciaire et les barrières institutionnelles qui réglementent l'accès aux différents programmes de réinsertion constituent deux composantes importantes qui permettent de comprendre le nivellement des conditions des deux groupes qui, pourtant, se différenciaient au point de départ des trajectoires.

Certains effets indirects du processus pénal et carcéral sont perceptibles dans nos analyses et permettent de mieux comprendre le maintien des répondantes dans des filières identiques à celles qui prévalaient déjà dans leur vie avant leur incarcération. Mis à part la suspension des conditions de survie qu'impose l'enfermement, la prison permet aux femmes autochtones préalablement insérées dans des réseaux illicites de maintenir ou reproduire des filières identiques dans lesquelles elles s'inséreront à leur sortie de prison. En fin de compte, le poids du pénal et du carcéral est important dans la mise en marge des répondantes autochtones. Mais il doit être relativisé dans la mesure où il est cumulé à des conditions et un contexte de vie qui existaient déjà avant la prise en charge pénale. 


\section{Références}

Affaires indiennes et du Nord Canada (2008). En ligne à URL: http://www. ainc-inac.gc.ca/index-fra.asp.

Azaola, E. (1996). Les femmes autochtones incarcérées au Mexique. Recherches amérindiennes au Québec, 26 (3-4), 75-82.

Balan, J., \& Jelin, E. (1980). La structure sociale dans la biographie personnelle. Cabiers internationaux de sociologie, 69, 269-289.

Bertaux, D. (1988). Fonctions diverses des récits de vie dans le processus de recherche. Sociétés, 18, 18-22.

Blanchard, B. (2002). La situation des mères incarcérées et de leurs enfants au Québec. Criminologie, 35 (2), 91-112.

Bourdieu, P. (1979). La distinction: Critique sociale du jugement. Paris: Minuit.

Braman, D. (2004). Doing Time on the Outside: Incarceration and Family Life in Urban America. USA: University of Michigan Press.

Brassard, R. (2006). L'expérience de l'enfermement carcéral des femmes autochtones au Québec. Revue Femmes et Droit/Canadian Journal Women and the Law, 17 (2), 311- 340.

Canada (2001). Les femmes autochtones: un portrait d'après le recensement de 1996. Section des statistiques sur les Premières Nations et le Nord, Ministère des Affaires indiennes et du Nord Canada.

Canada (2004). L'incarcération au Canada: État des données pour 2000-2001. Statistique Canada, Ottawa: Canada.

Canada (2008). Profil des enfants, adolescents et adultes autochtones 2006. Consulté le 20 mars 2009, http://www12.statcan.ca/censusrecensement/2006/ $\mathrm{dppd} / 89635 /$ index.cfm? Lang $=$ fra \&intro $=2$.

Castel, R. (1994). La dynamique des processus de marginalisation: de la vulnérabilité à la désaffiliation. Cabiers de recherche sociologique, 22, 11-27.

Chantraine, G. (2004). Par-delà les murs. Le Monde: PUF.

Combessie, Ph. (2004). Intégration sociale des anciens détenus. Analyse des logiques de la justice pénale et de leurs effets. In J. Poupart (Ed.), Au-delà du système pénal. L'intégration sociale et professionnelle des groupes judiciarisés et marginalisés (231-248). Québec: Presses de l'Université du Québec.

Commission royale sur les peuples du Canada. (1996). Par delà les divisions culturelles. Rapport sur les autochtones et la justice pénale au Canada, Ottawa, Canada.

Corbin, J., \& Strauss, A. L. (1990). Grounded Theory Research: Procedures, Canons and Evaluative Criteria. Qualitative Sociology, 13 (1), 3-21.

De Gaulejac, V., \& Léonetti, I. (1994). La lutte des places. Paris : Éditions Desclée de Brouwer.

Denzin, N. K. (1970). The Research Act: A Theoretical Introduction to Sociology Methods. San Francisco: McGraw-Hill.

Ditton, P. M. (1999). Mental Health and Treatment of Inmates and Probationers. Washington, D.C.: Department of Justice, Bureau of Justice Statistics, U.S. Department of Justice 
Dubec, B. (1982). Native Women and the Criminal Justice System: An Increasing Minority. Thunder Bay, Ontario: Native Women Association.

Dubet, F. (1994). Sociologie de l'expérience, Paris: Éditions du Seuil.

Enquêteur correctionnel (2007). Rapport annuel du Bureau de l'enquêteur correctionnel 2006-2007. Ottawa: Ministère des Travaux publics et Services gouvernementaux.

Ferraro, K., Johnson, J., Jorgensen, S., \& Bolton, F. (1983). Problems of Prisoner's Families: the Hidden Costs of Imprisonment. Journal of Family Issues, 4 (4), 575-591.

Frigon, S., \& Kerisit, M. (2000). Du corps des femmes: contrôles, surveillances et résistances. Ottawa: Les Presses de l'Université d'Ottawa.

Giddens, A. (1984). La constitution de la société, Paris: PUF.

Girard, R. (1972). La violence et le sacré. Paris: Grasset.

Grassian, S. (1983). Psychopathological Effects of Solitary Confinement. American Journal of Psychiatry, 140 (11), 1450-1454.

Grobsmith, E. S. (1995). Indians in Prison: Incarcerated Native Americans in Nebraska, Lincoln and London: University of Nebraska Press.

Grossman, M. G. (1992). Two Perspectives on Aboriginal Female Suicides in Custody. Canadian Journal of Criminology, 34 (3-4), 403-41.

Hamelin, M. (1989). Femmes et prisons, Montréal: Éditions du Méridien.

Hattem, T., \& Parent, C. (1982). Les effets négatifs d'un casier judiciaire au niveau de l'emploi. Les cahiers de l'École de criminologie, 8. Montréal: École de criminologie.

Jaccoud, M. (1992). Les femmes autochtones et la justice pénale. Criminologie, $25(1), 65-85$.

Jackson, M. (1999). Canadian Aboriginal Women and Their Criminality: The Case of Violence in the Context of Difference. The Australian and New Zealand Journal of Criminology, 32 (2), 197-208.

Johnson, H. (1986). Les femmes et la criminalité au Canada. Ottawa: Direction des programmes, Secrétariat du ministère, ministère du Solliciteur général du Canada.

Joly, D. \& Beckford, J. (2006). Race, Relations and Discrimination in Prison: The Case of Muslims in France and Britain. Journal of Immigrant and Refugee Studies, 4 (2), 1-30.

Kauffman, K. (2001). Mothers in Prison. Corrections Today, 63 (1), 62-65.

Kellerhals, J., Lazega, E., \& Troutot, P.-Y. (1983). Quelques notes sur l'utilisation interactive du récit de vie. Revue suisse de sociologie, 9 (1), 127-136.

Kreig, A. S. (2006). Aboriginal Incarceration: Health and Social Impacts. Medical Journal of Australia, 184 (10), 534-536.

Lainé, A. (1998). Faire de sa vie une bistoire: Théories et pratiques de l'bistoire de vie en formation. Paris: Desclée de Brouwer.

Laberge, D., \& Morin, D. (1997). L'incarcération comme mode de gestion de l'itinérance. Revue internationale de criminologie et de police technique, 50 (1), $30-53$. 
Laberge, D. Landreville, P., Morin, D., \& Casavant, L. (1998). Le rôle de la prison dans la production de l'itinérance. Cabier spécial du Collectif de Recherche sur l'itinérance. Montréal, Québec, Canada.

Landreville, P., Blankevoort, V. J., \& Pires A. P. (1981). Les coûts sociaux du système pénal. Montréal: Université de Montréal, Cahier de l'École de criminologie.

Landreville, P. (2002). L'impact des antécédents judiciaires sur la réinsertion dans le marché du travail. Conférence présentée au Colloque international francophone: Au-delà du système pénal es coûts sociaux du système pénal: L'intégration sociale et professionnelle des personnes judiciarisées. (Document inédit).

Landreville, P. (2004). Réinsertion dans le marché du travail. L'impact des antécédents judiciaires. In J. Poupart (Ed.), Au-delà du système pénal. L'intégration sociale et professionnelle des groupes judiciarisés et marginalisés (213-230). Québec, QC: Presses de 1'Université du Québec.

LaPrairie, C. (1996). Les services correctionnels pour Autochtones. Collection sur les Autochtones. Ministère du Solliciteur général du Canada, Approvisionnements et Services Canada.

LaPrairie, C. (1997). Reconstructing Theory: Explaining Aboriginal OverRepresentation in the Criminal Justice System in Canada. Australian and New Zealand Journal of Criminology, 30 (1), 39-54.

Liebling, A., \& Maruna, S. (2005). The Effects of Imprisonment. Oregon: Willan Publishing.

Marchetti, A.-M. (1996). Pauvreté et trajectoire carcérale. In C. Faugeron, A. Chauvrenet \& P. Combessi (Ed.), Approches de la prison, Perspectives criminologiques (177-194). Bruxelles: De Boeck.

Maruna, S., \& Immarigeon, R. (2004). After Crime and Punishment: Pathways to Offender Reintegration. Oregon: Willan Publishing.

McAll, C. (1995). Les murs de la cité: territoires d'exclusion et espaces de citoyenneté. Lien social et Politiques-RIAC, 34, 81-92.

Metcalf, H., Anderson, T., \& Rolfe, H. (2001). Barriers to Employment for Offenders and Ex-Offenders. A report of research carried out by the National Institute of Economic and Social Research on behalf of the Department for Work and Pensions, Norwich: Her Majesty's Stationary Office.

Monture-Angus, P. (2003). The Lived Experience of Discrimination: Aboriginal Women Who Are Federally Sentenced. Consulté le 13 janvier 2009, http://www.elizabethfry.ca/submissn/aborigin/1.htm.

Morin, D. (2002). Doing Time. In B. Schissel, \& C. Brooks (Eds.), Marginality and Condemnation: An Introduction to Critical Criminology (329-347). Halifax: Fernwood Press.

Ouimet. R. (1969). Rapport du comité canadien de la réforme pénale et correctionnelle. Ottawa, Ont.: Imprimeur de la Reine.

Paugam, S. (1996). L'exclusion: État des savoirs. Paris: La Découverte.

Petersilia, J. (2003). When Prisoners Come Home: Parole and Prisoner Reentry. England: Oxford University Press. 
Pires, A. (1983). Stigmate pénal et trajectoires sociales. Thèse de doctorat, École de criminologie, Université de Montréal, Montréal.

Plourde, C., Brochu, S., Couvrette, A., \& Gendron, A. (2007). Points de vue de femmes incarcérées dans des établissements fédéraux concernant les impacts du contexte carcéral sur leur trajectoire de consommation de drogues. Criminologie, 40 (1), 105-134.

Ross, L. (1998). Inventing the Savage: The Social Construction of Native American Criminality. Austin: University of Texas Press.

Sloop, J. M. (2006). The Cultural Prison: Discourse, Prisoners, and Punishment. University Alabama Press: New Edition.

Statistique Canada (2005). Femmes au Canada: rapport statistique fondé sur le sexe (5e édition). Ottawa: Canada: Statistique Canada.

Statistique Canada (2008). Peuples autochtones du Canada en 2006 : Inuits, Métis et Premières nations, Recensement de 2006 : faits saillants. En ligne: http://www12.statcan.ca/francais/census06/analysis/aboriginal/highlights.cfm

Sugar, F., \& Fox, L. (1990). Survey of Federally Sentenced Aboriginal Women in the Community. Canada: Native Women's Association of Canada.

Travis, J., \& Waul, M. (2003). Prisoners Once Removed: The Impact of Incarceration and Reentry on Children, Families, and Communities. Washington: The Urban Institute Press.

Trevethan, S., Auger S., Moore, J., MacDonald, M., \& Sinclair, J. (2002). The Effect of Family Disruption on Aboriginal and non-Aboriginal Inmates. Ottawa: Correctional Services Canada.

Woolredge, J. D., Masters, K. (1993). Confronting Problems Faced by Pregnant Inmates in State Prisons. Crime and Delinquency, 39 (2), 195-203.

Xiberras, M. (1998). Les théories de l'exclusion. Paris: Éditions Armand Colin.

Zambrowsky-Cross, S. (1986). Évaluation des besoins chez les femmes autochtones ayant ou risquant d'avoir des démêlés avec la justice dans la région de Montréal. Rapport pour spécialistes.

ABSTRACT - This article presents the results of a qualitative study on the effects of incarceration on the social exclusion in life stories of seven Aboriginal women in Québec. Findings indicate that, at the beginning of their life trajectory, three women evolved in a relatively stable family while four others lived in families experiencing multiple social problems. These group distinctions were practically maintained until initial contact with imprisonment, but grew increasingly blurred after the women's contact with criminal detention. In final analysis, the weight of incarceration is significant in the exclusion of Aboriginal women. However, it must be correlated to individual living conditions as well as particular life trajectories which precede the actual prisoning process. These results put in perspective the postulate on which the studies on the effects of incarceration are usually based.

KEYWORDS - Aboriginal, exclusion, Québec, prison, life trajectory, reintegration, women. 
RESUMEN - Este artículo presenta los resultados de un estudio cualitativo sobre los efectos del encarcelamiento en la trayectoria de vida de siete mujeres autóctonas de Quebec. Al inicio de su ciclo de vida, tres de las entrevistadas evolucionaron en un medio familiar relativamente estable y cuatro vivieron en un medio familiar con múltiples problemas sociales, diferencia que se mantiene prácticamente igual hasta el momento de la detención, para atenuarse paulatinamente luego de la experiencia de encarcelamiento. El estigma de los antecedentes penales y las reglas de acceso a los programas denominados de reinserción social resultan los efectos igualadores más importantes de las condiciones de vida de nuestro grupo de entrevistadas y contribuyen a igualar a la baja sus condiciones de vida. Así, los antecedentes penales contribuyen a excluirlas definitivamente del mercado laboral, dificultan su acceso a la vivienda y provocan su marginalización en sus comunidades de origen. El peso del encarcelamiento es, pues, importante en la marginalización de las mujeres autóctonas, pero se le debe evaluar junto con otras condiciones y el contexto de vida que le precede.

PALABRAS CLAVE - Autóctono, exclusión, mujeres, prisión, Quebec, reinserción, trayectoria de vida. 